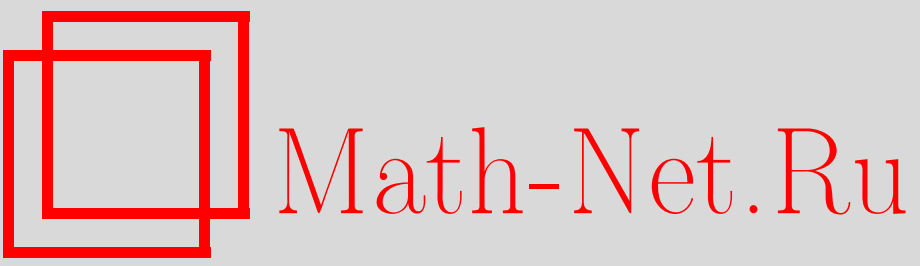

В. В. Кулямин, Образы градуированных многочленов в кольцах матриц над конечными групповыми алгебрами, УМН, 2000, том 55, выпуск 2, 141-142

DOI: https://doi.org/10.4213/rm278

Использование Общероссийского математического портала Math-Net.Ru подразумевает, что вы прочитали и согласны с пользовательским соглашением

http://www.mathnet.ru/rus/agreement

Параметры загрузки:

IP: 54.81 .137 .203

26 апреля 2023 г., 16:25:34 


\title{
ОБРАЗЫ ГРАДУИРОВАННЫХ МНОГОЧЛЕНОВ В КОЛЬЦАХ МАТРИЦ НАД КОНЕЧНЫМИ ГРУППОВЫМИ АЛГЕБРАМИ
}

\author{
В.В. Кулямин
}

1. Введение. В этой работе изучаются образы многочленов от некоммутирующих переменных в кольце матриц над конечной коммутативной групповой алгеброй, рассматриваемой как градуированное кольцо. Основная цель работы - попытаться перенести на градуированньй случай следующее утверждение (см. [1]).

ТеОРема 1. Пусть $R=M_{n}(\mathbb{F})$ - кольцо матрич размера $n \times n$ над конечным полем $\mathbb{F}$. множество $A \subseteq R$ является образом многочлена от некоммутирующих переменных с нулевым свободным членом тогда и только тогда, когда $0 \in A$ и $\alpha A \alpha^{-1} \subseteq A$ для всех обратимых матрии $\alpha$ из $R$.

В качестве основного кольца у нас будет выступать конечная коммутативная групповая алгебра с естественной градуировкой - такие градуированные кольца ближе всего по своим свойствам к полям. Введем ряд понятий, которые потребуются для формулировки основного резултата данной работы.

Для кольца $\mathbb{K}$, градуированного по полугруппе $G$, рассмотрим алгебру $G$-градуированных многочленов над $\mathbb{K}$ - свободную ассоциативную алгебру $\mathbb{K}\{X\}$, порожденную $G$-градуированныцм множеством $X$, т.е. множеством, представленным в виде дизъюнктного объединения

$$
X=\bigsqcup_{g \in G} X_{g}
$$

компонент $X_{g}$, каждая из которых является счетным множеством $X_{g}=\left\{x_{g 0}, x_{g 1}, \ldots\right\}$. Далее мы будем обозначать эту алгебру как $\mathscr{K}^{G}$, а подалгебру многочленов с нулевым свободным членом в ней как $\mathscr{K}_{0}^{G}$.

Обозначим также через $\operatorname{Hom}_{\mathbb{K}}^{G}(R, S)$ множество гомоморфизмов $G$-градуированной $\mathbb{K}$-алгебры $R$ в $G$-градуированную $\mathbb{K}$-алгебру $S$, сохраняющих градуировку, т.е. удовлетворяющих условию

$$
\forall g \in G \quad \varphi\left(R_{g}\right) \subseteq S_{g}
$$

Будем назьвать их далее $G$-градуированными гомоморфизмами.

Образом многочлена $f$ из $\mathscr{K}^{G}$ в $G$-градуированной алгебре $R$ назовем множество

$$
\operatorname{Im} f=\left\{a \in R: \exists \varphi \in \operatorname{Hom}_{\mathbb{K}}^{G}(\mathbb{K}\{X\}, R) \varphi(f)=a\right\} .
$$

Это определение означает, что, при взятии образа градуированного многочлена, мы подставляем в него вместо переменных $x_{g i}$ всевозможные однородные элементы степени $g$ из $R$.

Далее для $a \in R$ будем обозначать через $a_{g}$ его однородную компоненту степени $g$, а для $A \subseteq R$ через $A_{g}$ - его проекцию на $R_{g}$.

Пусть $e$ - единица полугруппы $G$. Множество матриц $A$ над градуированным по $G$ кольцом $\mathbb{K}$, удовлетворяющее условию $\alpha A \alpha^{-1} \subseteq A$ для всякой обратимой однородной степени $е$ матрицы $\alpha$, будем называть самоподобныц.

Теперь сформулируем основной результат этой работы.

Теорема 2. Пусть $R=M_{n}(\mathbb{K})$ - кольчо матриц размера $n \times n$ над конечной коммутативной групповой алгеброй $\mathbb{K}=\mathbb{F}(G)$ с естественной градуировкой. Однородное множество $A \subseteq R$ является образом $G$-градуированного многочлена из $\mathscr{K}_{0}^{G}$ тогда и только тогда, когда $0 \in A$ и А самоподобно.

2. Доказательство основного результата. Мы всюду будем придерживаться обозначений, введенных в формулировке теоремы 2.

Очевидно, что однородное множество, являющееся образом градуированного многочлена с нулевым свободным членом, содержит 0 . Пусть также $a=f\left(a_{1}, \ldots, a_{m}\right)$, где $a, a_{1}, \ldots, a_{m} \in R$ и $\alpha \in R$ - однородная обратимая матрица. Тогда

$$
\alpha a \alpha^{-1}=\alpha f\left(a_{1}, \ldots, a_{m}\right) \alpha^{-1}=f\left(\alpha a_{1} \alpha^{-1}, \ldots, \alpha a_{m} \alpha^{-1}\right) .
$$


Последнее выражение является матрицей из $\operatorname{Im} f$, поскольку степень однородности $\alpha a_{i} \alpha^{-1}$ совпадает со степенью однородности $a_{i}$. Таким образом, необходимость в теореме 2 доказана.

Далее нам понадобится следующее утверждение, которое легко извлечь из доказательства теоремы 1 в [1].

Лемма 3. Для самоподобного множества А матрии над конечным полем существует многочлен от некоммутирующих переменных $\chi_{A}\left(x, x_{1}, \ldots, x_{m}\right)$, принимающий только значения 0 и 1 , и такой, что

$$
\begin{aligned}
& a \notin A \Rightarrow \forall a_{1}, \ldots, a_{m} \in R \quad \chi_{A}\left(a, a_{1}, \ldots, a_{m}\right)=0 \\
& a \in A \Rightarrow \exists a_{1}, \ldots, a_{m} \in R \quad \chi_{A}\left(a, a_{1}, \ldots, a_{m}\right)=1 .
\end{aligned}
$$

Многочлен, обладающий описанными свойствами, будем называть индикатором множества $A$.

Под классом подобия в $R$ будем понимать минимальное непустое самоподобное множество. Назовем класс подобия матриц $C \subseteq R$ разложимым, если он является суммой своих проекций на однородные компоненты $R$ (если $G=\left\{g_{1}, \ldots, g_{k}\right\}$ и $a_{1}, \ldots, a_{k} \in C$, то $\sum_{g \in G} a_{i g_{i}} \in C$ ). Очевидно, что класс подобия, состоящий из однородных матриц одной степени однородности, является разложимым - все его проекции, кроме одной, равны $\{0\}$.

Лемма 4. Всякий разложимый класс подобия матрии $C$ над конечной коммутативной групповой алгеброй $\mathbb{K}=\mathbb{F}(G)$ является образом градуированного многочлена из $\mathscr{K}_{0}^{G}$.

ДокаЗАТЕЛЬСтво. Рассмотрим множество однородных компонент степени $g_{i}$ элементов класса $C$. Легко показать, что оно представляет собой некоторое самоподобное множество матриц $C_{\left(g_{i}\right)}$ над $\mathbb{F}$, умноженное на $g_{i}$. По лемме $3 C_{\left(g_{i}\right)}$ имеет индикатор $\chi_{i}\left(x_{i}, x_{i 1}, \ldots, x_{i m_{i}}\right)$ в кольце матриц над $\mathbb{F}$. Рассмотрим многочлен

$$
h\left(y_{1}, \ldots, y_{k}, x_{11}, \ldots, x_{k m_{k}}\right)=\left(g_{1} y_{1}+\cdots+g_{k} y_{k}\right) \prod_{i \leqslant k} \chi_{i}\left(y_{i}, x_{i 1}, \ldots, x_{i m_{i}}\right),
$$

в котором степень однородности переменных $y_{i}, x_{i 1}, \ldots, x_{i m_{i}}$ равна $e$ - единице $G$. Ясно, что он лежит в $\mathscr{K}_{0}^{G}$. Очевидно также, что если значение $y_{i}$ для какого-нибудь $i$ не лежит в $C_{\left(g_{i}\right)}$, то $h$ принимает значение 0 , если же для всякого $i$ значение $y_{i}$ - элемент $C_{\left(g_{i}\right)}$, то значение $h$ равно сумме произведений их значений на соответствующие элементы группы $G$ и, следовательно, лежит в $C$. Беря в качестве $g_{i} y_{i}$ однородные компоненты произвольного элемента $C$, легко показать, что он лежит в образе многочлена $h$.

ЛЕмма 5. Если $E\left(z_{1}, \ldots, z_{l}\right)$ - градуированный многочлен в $R$, принимающий только значения 0 и 1 но не являющийся тождеством, то образом многочлена

$$
\begin{aligned}
f\left(x_{1}, \ldots, x_{m}, y_{1}, \ldots, y_{s}, z_{1}, \ldots, z_{l}\right) \\
\quad=E\left(z_{1}, \ldots, z_{l}\right) h\left(x_{1}, \ldots, x_{m}\right)+\left(1-E\left(z_{1}, \ldots, z_{l}\right)\right) p\left(y_{1}, \ldots, y_{s}\right)
\end{aligned}
$$

является объединение образов $h$ и

Доказательство этого утверждения очевидно. Соединяя его с уже отмеченными фактами, легко видеть, что любое содержащее 0 объединение разложимых классов подобия матриц над конечной коммутативной групповой алгеброй является образом градуированного многочлена с нулевым свободным членом, в частности, таковыми являются однородные самоподобные содержащие 0 подмножества, что и завершает доказательство теоремы 2.

\section{СПИСОК ЛИТЕРАТУРЫ}

[1] Chuang C.-L. // Proc. Amer. Math. Soc. 1990. V. 110. № 2. P. 293-302.

ИСП РАН

E-mail: kuliamin@ispras.ru
Принято редколлегией 26.01 .2000 